\title{
Investigating the response of skeletal muscle to prosthesis-related loading conditions: An ex vivo animal model \\ Alternative Title: Development of an ex vivo animal model to study the response of skeletal muscle to prosthesis-related loading conditions
}

\author{
Marisa Graser ${ }^{1}$, Arjan Buis ${ }^{1}$ \\ 1 University of Strathclyde
}

\section{BACKGROUND (70 words)}

The use of lower-limb prosthetics puts the soft tissue of the residuum, including the muscle envelop, under constant physical stress. To adapt to this unphysiological mechanical loading, the muscles need to maintain the balance between tissue damage and regenerative processes. However, in extreme cases of overload or with repeated impact, this balance may be disturbed [1], potentially leading to residual limb pain and Deep Tissue Injuries [2].

\section{AIM (30 words)}

A new ex vivo model of rat skeletal muscle tissue was developed to quantify cellular damage from different prosthetic-related loading protocols. Experimental procedures and the relevance of the results for prosthetic research and practice are discussed.

\section{METHOD (100 words)}

In a number of preliminary experiments, excised soleus and extensor digitorum longus muscles of Sprague Dawley rats were subjected to transverse compressive loading. The following preparation of the specimen for imaging included standard histological procedures as well as tissue clearing. Subsequently, the samples were visualised through optical imaging and vibrational spectroscopy to evaluate the extend of cellular damage. Complementing the ex vivo experiments, FE models were created to relate the observed cellular damage to the internal deformation of the specimen during the loading protocols.

\section{RESULTS (130 words, incl. figure/table)}

During the experimental time frame of maximum $3 \mathrm{~h}$, the muscle samples showed minor loss in viability due to insufficient oxygen supply. However, by comparison with control samples, the extend of mechanical damage to the cells could be distinguished. Both standard histology as well as the tissue clearing protocol induced minor changes to the samples, like shrinkage. The influence of these artefacts was minimised through an appropriate setup and careful practice. With regards to imaging, all types of microscopy, including epifluorescent, confocal, and Raman microscopy, were able to produce high quality images. The visibly damaged areas correlated well with predicted areas of high load of the FE models.

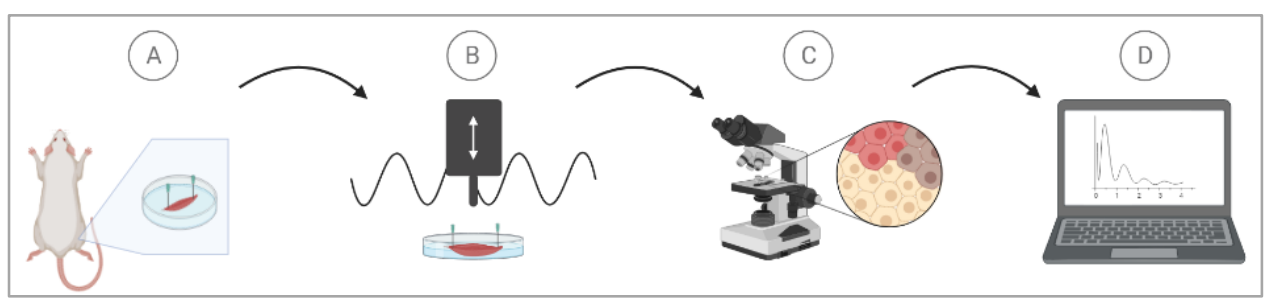

Figure 1. Ex vivo animal model. A: Skeletal muscle dissection; B: Mechanical loading protocol; C: Image analysis for cell damage; $\mathrm{D}$ : Computational modelling combining data on cell damage with internal loading conditions

\section{DISCUSSION AND CONCLUSION (70 words)}

Our preliminary studies present an ex vivo animal model and experimental procedures that are suitable to quantify cellular damage from prosthesis-related loading conditions on skeletal muscle. Looking at this microscale will provide important insights into the adaptive capabilities of skeletal muscle. This can provide the basis for further research into the role of soft tissue deformation in limb pain and ulcer formation. Additionally, it can inform future discussions on socket design and fit.

\section{REFERENCES (50 words)}

1. MJ Mueller; 2002; Phys Ther.

2. M Graser; 2020; BMC BiomEng. 